\title{
ANÁLISIS ECONÓMICO DE LA UTILIZACIÓN DE INOCULANTE BIOLÓGICO (Rhizobium sp.) EN FRIJOL COMÚN, EN LA REGIÓN BRUNCA, COSTA RICA1
}

\author{
Guillermo Flores 2 , Juan Carlos Hernández ${ }^{2}$, Miguel Acosta ${ }^{3}$, Mario Montero ${ }^{4}$
}

\begin{abstract}
RESUMEN
Análisis económico de la utilización de inoculante biológico (Rhizobium sp.) en frijol común, en la Región Brunca, Costa Rica. El estudio se realizó en dos zonas de la Región Brunca con inoculante biológico a base de bacterias del género Rhizobium sp. con el propósito de disminuir la utilización de fertilizantes químicos. Se evaluaron cuatro tratamientos: Testigo absoluto; Inoculante $(0,7 \mathrm{~kg} / \mathrm{ha})$; Inoculante $(0,7 \mathrm{~kg} / \mathrm{ha})+$ Fertilizante $10-30-10(63 \mathrm{~kg} / \mathrm{ha})$ y Fertilizante 10-30-10 (125 kg/ha); a nivel de finca de agricultor a una densidad de siembra de 30-32 kg semilla/ha. La técnica utilizada para realizar el análisis fue la de presupuesto parcial y un análisis de Dominancia (CIMMYT, 1988), para determinar beneficio neto y tasa marginal de retorno. En la zona de Changuena, Buenos Aires el uso de solo inoculante presentó el mayor beneficio neto y una tasa de retorno marginal del $388 \%$. Para la zona Pejibaye de Pérez Zeledón la utilización de solo fertilizante produjo el mayor beneficio neto y una tasa de retorno marginal de $621 \%$, inoculante + fertilizante $814 \%$ y solo inoculante $173 \%$. El uso de inoculante biológico fue una práctica económicamente rentable para los agricultores.
\end{abstract}

\begin{abstract}
Economic analysis of the use of biological inoculants in Common Bean, in two zones of the Brunca Region, Costa Rica. This study was carried out in two zones of the Brunca Region using a biological inoculants of the Rhizobium sp bacteria. The objective was to reduced the chemical fertilizer utilization. Four treatments were evaluated: Absolute control; Inoculants $(0,7 \mathrm{~kg} / \mathrm{ha})$; Inoculants $(0,7 \mathrm{~kg} / \mathrm{ha})+$ Fertilizer $10-30-10(63 \mathrm{~kg} / \mathrm{ha}$ is $)$ and Fertilizer10-30-10 (125 kg/ha) in property of farmer at a density of 30-32 kg seed/ha. A partial budget and an analysis of Dominance (CIMMYT, 1988) were used to carry out the economic analysis determining net profit and marginal rate of return. In the zone of Changuena, Buenos Aires the use of single inoculants presented the higher net profit and a rate of marginal return of $388 \%$. For the zone of Pejibaye de Pérez Zeledón the use of single fertilizer obtained the higher net profit and a rate of marginal return of $621 \%$, inoculants+ fertilizer $814 \%$ and single inoculants $173 \%$. The use of biological inoculants was a practice economically profitable for the farmers
\end{abstract}

\section{INTRODUCCIÓN}

El cultivo de frijol en Costa Rica es una actividad de gran importancia, para pequeños y medianos productores. El 26\% del área nacional sembrada de frijol corresponde a la región Brunca.

El uso de inoculantes biológicos es una práctica que recientemente se ha venido utilizando en nuestro país especialmente en las zonas de mayor producción de frijol (Región Huetar Norte y Región Brunca); con el propósito de disminuir la utilización de fertilizantes químicos, ayudando a preservar el ambiente y reducir los costos de producción.

Se han realizado gran cantidad de experimentos que han demostrado que es posible lograr incrementos en los rendimientos que varían entre un $20 \%$ y $60 \%$

\footnotetext{
1 Trabajo presentado al Comité Organizador del PCCMCA, Nicaragua, 1998. Financiado parcialmente por PROFRIJOL.

2 Dirección Investigaciones Agropecuarias, Ministerio de Agricultura y Ganadería, Apdo. Postal 10094-1000. San José, Costa Rica.

3 Coordinador Granos Básicos. Dirección Regional Brunca. MAG.

4 Extensionistas. Agencia de Pejibaye y Agencia de Potrero Grande, respectivamente.
} 
cuando se inocula con cepas altamente efectivas en el proceso de fijación de N (Acuña, O.,1996). Por lo tanto es importante determinar a nivel de finca, la factibilidad económica de esta práctica y la aceptación de la misma por parte de los agricultores.

Para el desarrollo de nódulos y la fijación de N, se ha determinado una correlación directa con la relación $\mathrm{C}: \mathrm{N}$ en el tejido de las plantas, donde relaciones altas favorecen y relaciones bajas inhiben la nodulación (Acuña, O., 1996). También que existen otros nutrimentos que juegan un papel muy importante en la fijación de nitrógeno entre estos podemos destacar el: P, K, Ca, B, $\mathrm{Mg}, \mathrm{S}, \mathrm{Mo}, \mathrm{Cu}, \mathrm{Zn}, \mathrm{Co}$, donde tiene especial atención el fósforo, de allí que se estén seleccionando cepas que puedan desarrollarse a bajos niveles de este elemento.

Por su parte Vargas y Acuña (1990) manifiestan que es recomendable aplicar una pequeña cantidad de fertilizante nitrogenado ( $30 \mathrm{~kg} / \mathrm{ha})$ a la hora de la siembra, para suplir la necesidad de la planta por este elemento durante los estados iniciales de la infección y crecimiento nodular, especialmente cuando el crecimiento nodular no se ha establecido.

En la actualidad el principal desafío que afronta el cultivo de frijol es el proceso de liberalización comercial e integración centroamericana, dado que el productor nacional debe estar en capacidad de tener un producto de calidad a precio competitivo para conservar el mercado local y competente en el mercado externo.

El objetivo de este análisis es determinar el comportamiento económico de la utilización de inoculantes biológicos en frijol en comparación con la fertilización química.

\section{MATERIALES Y MÉTODOS}

El estudio se llevó a cabo en dos zonas de la región Brunca (Cuadro 1). Para fines de análisis, se agruparon las dos parcelas de Changuena para formar una zona.
Debido a la similitud de ambiente, suelo y topografía que existen entre Concepción y Veracruz, se tomará ésta como la segunda zona de estudio.

Los insumos de producción fueron suministrados por los técnicos de las Agencias de Extensión de Pejibaye y Potrero Grande. En la ejecución de las labores de campo participaron conjuntamente los técnicos de dichas agencias y productores; siendo éstos últimos los que dieron seguimiento al estudio y los que suministraron la información primaria.

Se evaluaron los cultivares criollos de grano rojo Vaina Blanca en Changuena y Saca Pobres en Concepción y Veracruz; a una densidad de siembra de 30-32 kg semilla/ha.

\section{Tratamientos:}

Se evaluaron cuatro tratamientos, los cuales se describen a continuación: Testigo absoluto; Inoculante $(0,7$ $\mathrm{kg} / \mathrm{ha})$; Inoculante + Fertilizante $(0,7 \mathrm{~kg} / \mathrm{ha}+63 \mathrm{~kg} / \mathrm{ha}$ de 10-30-10); Fertilizante (125 kg/ha de 10-30-10).

El período de análisis abarcó la segunda época de siembra durante el período octubre 1997 a febrero de 1998.

Los rendimientos obtenidos por los distintos tratamientos durante todo el período de análisis, así como los costos y los ingresos fueron sometidos a un análisis de dominancia (CIMMYT, 1988). Este análisis consistió en determinar el beneficio neto y la tasa marginal de retorno de cada tratamiento para clasificarlos como dominados y no dominados, donde estos últimos fueron los que presentaron los mayores beneficios a costos menores.

Estos criterios de selección de los diferentes tratamientos fue el que produjo el mayor beneficio neto a una tasa marginal de retorno mínima aceptable (100\%) de acuerdo al costo de oportunidad del dinero y la aversión al riesgo.

Cuadro 1. Productor y área sembrada por parcela según localidad. Región Brunca. Costa Rica. 1997-1998.

\begin{tabular}{clllc}
\hline $\mathbf{N}^{\mathbf{0}}$ & \multicolumn{1}{c}{ Productor } & \multicolumn{1}{c}{ Localidad } & \multicolumn{1}{c}{ Cantón } & Área/parcela \\
\hline 1 & Daniel Alvarado & Changuena & Buenos Aires & $225 \mathrm{~m}^{2}$ \\
2 & Asdrubal Alvarado & Changuena & Buenos Aires & $225 \mathrm{~m}^{2}$ \\
3 & Marvin Mora & Concepción de Pilas & Buenos Aires & $400 \mathrm{~m}^{2}$ \\
4 & Ulises Marín & Veracruz & Pérez Zeledón & $400 \mathrm{~m}^{2}$ \\
\hline
\end{tabular}

Fuente: Área de Socioeconomia DIA-MAG con base en información suministrada por los agricultores. 1998. 
El análisis se llevo a cabo con los valores promedios ajustados de los rendimientos, costos e ingresos obtenidos según tratamientos a través de todo el ciclo del cultivo.

El análisis de dominancia se basó en la técnica de presupuesto parcial, por lo que para obtener el costo variable únicamente se incluyeron aquellos costos que variaron entre tratamientos, es decir, mano de obra para aplicar el fertilizante y el inoculante; también el costo de los insumos (inoculante y fertilizante).

El beneficio neto constituyó por lo tanto la diferencia entre los ingresos totales y los costos variables totales. La tasa marginal de retorno se calculó con base en el coeficiente obtenido de la diferencia del beneficio neto y el costo variable entre tratamientos de un beneficio neto inferior a otro mayor.

\section{RESULTADOS Y DISCUSIÓN}

Las siembras que se realizaron a finales del periodo 1997 y principios de 1998 a nivel comercial, en las diversas localidades en estudio fueron afectadas por el fenómeno del niño, causando a finales de 1997 pérdidas por exceso de lluvias en la Región Brunca. Dichas pérdidas se estimaron en un $30 \%$ del área total sembrada en la región.

De acuerdo al análisis de dominancia para la zona de Changuena, Buenos Aires (Cuadro 2) los tratamientos de sólo fertilizante químico y fertilizante + inoculante mostraron dominancia. La utilización de sólo inoculante no presentó dominancia y presentó un beneficio neto de $\varnothing 198089.5$, con una tasa marginal de retorno de $388 \%$; en relación al testigo absoluto.
Lo anterior se puede apreciar mejor en la Figura 1 en donde los beneficios netos aumentan al utilizar inoculante, mostrando para los productores de la zona de Changuena que la utilización del inoculante biológico es una nueva alternativa dentro del manejo agronómico de su parcela.

Por otra parte, el análisis de dominancia efectuado en la zona de Concepción y Veracruz (Cuadro 3), no mostró ninguna dominancia entre los tratamientos. La utilización de sólo fertilizante obtuvo el mayor beneficio neto y una tasa de retorno de $621 \%$. El segundo termino fue el inoculante + fertilizante con un beneficio neto un poco inferior y una tasa de retorno mucho mayor que el anterior (814\%). Ambos tratamientos superaron al inoculante solo con una tasa de retorno marginal de $173 \%$.

En la Figura 2 se observa el comportamiento de cada tratamiento destacándose como el mejor tratamiento, la utilización de solo fertilizante químico para la zona de Concepción y Veracruz.

Los suelos de la zona de Veracruz y Concepción son del orden Inceptisol y Ultisol, de topografía quebrada, con un manejo intensivo de producción de granos básicos a través de los años, que ha ocasionado una disminución en la fertilidad química de los mismos. En esta zona la respuesta por parte de la planta al uso de inoculante fue mínima, debido principalmente a falta de nutrimentos y desbalances de los mismos, por lo que la planta hace uso de la fuente mineral artificial (fertilizante químico).

Caso contrario sucedió en la zona de Changuena en donde los suelos son de topografía quebrada pero con un uso menos intensivo y una mayor cobertura del

Cuadro 2. Ingreso total, costo variable, beneficio neto y tasa retorno marginal (TRM), de la utilización de inoculantes biológicos para Vaina Blanca de acuerdo a su dominancia. Changuena, Costa Rica. 1997-1998. ${ }^{1}$

\begin{tabular}{lcccccc}
\hline Tratamiento & $\begin{array}{c}\text { Rend. Prom. } \\
(\mathbf{k g} / \mathbf{h a})\end{array}$ & $\begin{array}{c}\text { Rend. Prom. } \\
\text { Ajustada } \\
(\mathbf{k g} / \mathbf{h a})^{\mathbf{2}}\end{array}$ & $\begin{array}{c}\text { Beneficio Bruto } \\
(\mathbf{(} / \mathbf{h a})^{\mathbf{3}}\end{array}$ & $\begin{array}{c}\text { Costo Variable } \\
(\boldsymbol{\phi} / \mathbf{h a})\end{array}$ & $\begin{array}{c}\text { Beneficio } \\
\text { Neto }(\boldsymbol{\phi} / \mathbf{h a})\end{array}$ & $\begin{array}{c}\text { Tasa Ret. Marg. } \\
\text { TRM }(\%)\end{array}$ \\
\hline $\begin{array}{l}\text { No Dominado } \\
\text { Testigo }\end{array}$ & 1134 & 1021 & 183780 & 0 & 183780 & - \\
$\begin{array}{l}\text { Inoculante } \\
\text { Dominado }\end{array}$ & 1245 & 1121 & 201780 & 3690.5 & 198089.5 & 388 \\
$\begin{array}{l}\text { Inoculante + Fert. } \\
\text { Fertilizante }\end{array}$ & 1245 & 1121 & 201780 & 11228 & 190552 & - \\
\hline
\end{tabular}

Fuente: Área de Socioeconomia DIA-MAG con base en información suministrada por los agricultores. 1998.

1 US $\$=\varnothing 245,66$.

2 Rendimiento Ajustado en un $10 \%$ del rendimiento total.

3 Precio Venta $=\not 180 / \mathrm{kg}$. 


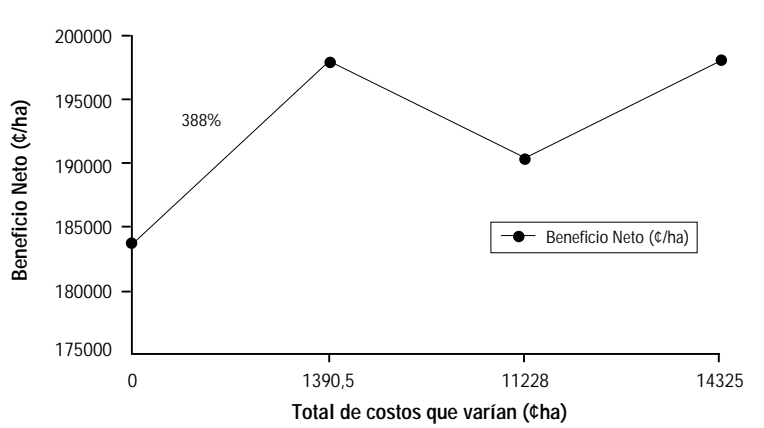

Figura 1. Comportamiento de la Curva de Beneficio Neto en ensayo de inoculantes, cultivar Vaina Blanca. Changuena, Buenos Aires. Región Brunca. 1998.

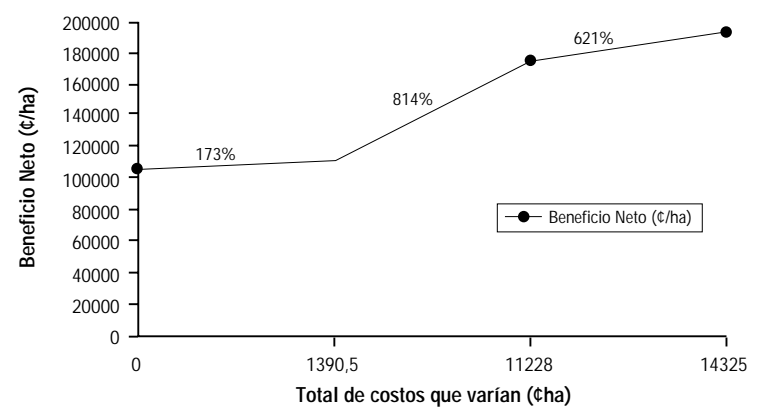

Figura 2. Comportamiento de la Curva de Beneficio Neto en ensayo de inoculantes, cultivar Saca Pobres, Concepción y Veracruz, Pérez Zeledón. Región Brunca. 1998.

Cuadro 3. Ingreso total, costo variable, beneficio neto y tasa retorno marginal (TRM), de la utilización de inoculantes biológicos para Saca Pobres de acuerdo a su dominancia. Concepción y Veracruz, Costa Rica. 1997-1998. 1

\begin{tabular}{lcccrrr}
\hline Tratamiento & $\begin{array}{c}\text { Rend. Prom. } \\
(\mathbf{k g} / \mathbf{h a})\end{array}$ & $\begin{array}{c}\text { Rend. Prom. } \\
\mathbf{A j u s t a d a}(\mathbf{k g} / \mathbf{h a}) \mathbf{2}\end{array}$ & $\begin{array}{c}\text { Beneficio Bruto } \\
(\mathbf{c} / \mathbf{h a}) \mathbf{3}\end{array}$ & $\begin{array}{c}\text { Costo Variable } \\
(\mathbf{c} / \mathbf{h a})\end{array}$ & $\begin{array}{c}\text { Beneficio Neto } \\
(\mathbf{c} / \mathbf{h a})\end{array}$ & $\begin{array}{c}\text { Tasa Ret. Marg. } \\
\mathbf{T R M}(\boldsymbol{\%})\end{array}$ \\
\hline No Dominado & & & & & & \\
Testigo & 650 & 585 & 105300 & 0 & 105300 & - \\
Inoculante & 713 & 641 & 115380 & 3690,5 & 111689,5 & 173 \\
Inoculante + Fert. & 1138 & 1024 & 184320 & 11228 & 173092 & 814 \\
Fertilizante & 1275 & 1148 & 206640 & 14325 & 192315 & 621 \\
\hline
\end{tabular}

Fuente: Área de Socioeconomia DIA-MAG con base en información suministrada por los agricultores. 1998.

1 UUS $\$=\varnothing 245,66$.

2 Rendimiento Ajustado en un $10 \%$ del rendimiento total.

3 Precio Venta $=\varnothing 180 / \mathrm{kg}$.

suelo debido a que históricamente se han realizados siembras de frijol tapado; lo que ha permitido mantener mejores valores de materia orgánica, macronutrientes y $\mathrm{pH}$, lo que favorece el desarrollo inicial del cultivo y por ende una mejor utilización por parte de la planta del inoculante biológico.

Estas diferencias en cuanto a condición de suelo y manejo de cultivo por parte de los agricultores de ambas zonas, podrían explicar en parte los resultados obtenidos.

Los datos obtenidos del análisis económico son preliminares debido a que las parcelas de investigación fueron afectadas por condiciones anormales de precipitación causadas por el fenómeno de El NIÑO. No obstante con la información que se posee se puede concluir.

En la zona de Changuena hubo una respuesta económica positiva a la utilización de inoculante biológico con un beneficio neto de $\varnothing 198.090$, lo que representa una nueva alternativa de manejo para los productores de esta zona.
Para la zona de Concepción y Veracruz no hubo una respuesta económica positiva a la utilización de inoculante biológico. Siendo la fertilización química la que obtuvo el mayor beneficio neto $\varnothing 192.315$, seguido de fertilización química + inoculante biológico con $\varnothing$ 173.092 de beneficio neto y por último el inoculante biológico con un beneficio neto de $\varnothing 111.690$.

Se sugiere la realización de un nuevo estudio bajo las condiciones normales de ambiente en ambas zonas con la finalidad de darle seguimiento a la respuesta de la utilización de inoculante biológico.

\section{LITERATURA CITADA}

ACUÑA, O. 1996. Manejo y tecnología de la fijación biológica de nitrógeno en leguminosas de importancia agrícola. Memoria Suelos. Congreso Nacional Agronómico y de Recursos Naturales. San José. Costa Rica. 227 p.

CENTRO INTERNACIONAL PARA EL MEJORAMIENTO DE MAÍZ Y TRIGO (CIMMYT). 1988. La formulación 
de recomendaciones a partir de los datos agronómicos: Un manual metodológico de evaluación económica. Rev. México D.F., México, CIMMYT. 79 p.

CONSEJO NACIONAL DE PRODUCCIÓN. (CNP). 1997. Producción de Granos Básicos. Dirección de Mercadeo y Agroindustria. Sistemas de información de mercados. San José, Costa Rica. p. 214.

SALAZAR, J. 1995. Programa Nacional de Frijol. Consejo Nacional de Producción (CNP). San José, Costa Rica. p. 22.
SECRETARIA EJECUTIVA DE PLANIFICACIÓN SECTORIAL AGROPECUARIA (SEPSA). 1993. Diagnóstico del Sector Agropecuario. Tema 1. San José, Costa Rica. $200 \mathrm{p}$.

VARGAS, R; ACUÑA, O. 1990. Respuesta de dos variedades de Phaseolus vulgaris a la inoculación y a la fertilización con $\mathrm{N}$ y Mo en un Inceptisol de Upala, Alajuela. Agronomía Costarricense 14(1):93-98. 